\title{
On the volume of unit vector fields on spaces of constant sectional curvature
}

Fabiano B. Brito, Pablo M. Chacón and A. M. Naveira

\begin{abstract}
A unit vector field $X$ on a Riemannian manifold determines a submanifold in the unit tangent bundle. The volume of $X$ is the volume of this submanifold for the induced Sasaki metric. It is known that the parallel fields are the trivial minima.

In this paper, we obtain a lower bound for the volume in terms of the integrals of the $2 i$-symmetric functions of the second fundamental form of the orthogonal distribution to the field $X$.

In the spheres $\mathbf{S}^{2 k+1}$, this lower bound is independent of $X$. Consequently, the volume of a unit vector field on an odd-sphere is always greater than the volume of the radial field. The main theorem on volumes is applied also to hyperbolic compact spaces, giving a non-trivial lower bound of the volume of unit fields.
\end{abstract}

Mathematics Subject Classification (2000). 53C20, 58E35.

Keywords. Unit vector fields, Sasaki metric, volume functional, spaces forms.

\section{Introduction}

Let $M^{n}$ be a closed oriented Riemannian manifold and $X$ a unit vector field defined on $M$. The volume of $X$ was defined in [GZ] by

$$
\operatorname{vol}(X)=\operatorname{vol}(X(M)),
$$

where $X: M \rightarrow T^{1} M$ is seen as a smooth section on the unit tangent bundle $T^{1} M$, endowed with the Sasaki metric, and $\operatorname{vol}(X(M))$ is the volume of the submanifold $X(M) \subset T^{1} M$.

A metric formula for the volume of a unit vector field $X$ is given by (see [GZ] and $[\mathrm{J}])$

$$
\operatorname{vol}(X)=\int_{M} \sqrt{\operatorname{det}\left(\operatorname{Id}+(\nabla X)^{t}(\nabla X)\right)} \nu=
$$

First author was supported by CNPq (Brazil) Grant No. 301207/80 and by Fapesp (Brazil) Proj. Tem. No. 1999/02684-5. Second and third authors are partially supported by DGI (Spain) Grant No. BFM 2001-3548 and CCEGV (Spain) Grant No. GR00-52. During the elaboration of this paper second author was also partially supported by Fapesp (Brazil). 


$$
\begin{aligned}
= & \int_{M}\left(1+\sum_{a=1}^{n}\left\|\nabla_{e_{a}} X\right\|^{2}+\sum_{a<b}\left\|\nabla_{e_{a}} X \wedge \nabla_{e_{b}} X\right\|^{2}+\ldots\right. \\
& \left.\ldots+\sum_{a_{1}<\cdots<a_{n-1}}\left\|\nabla_{e_{a_{1}}} X \wedge \cdots \wedge \nabla_{e_{a_{n-1}}} X\right\|^{2}\right)^{\frac{1}{2}} \nu
\end{aligned}
$$

where $n=\operatorname{dim}(M), \nu$ is the volume form and $\left\{e_{i}\right\}_{i=1}^{n}$ is an orthonormal local frame. It follows from that formula that $\operatorname{vol}(X) \geq \operatorname{vol}(M)$ and equality holds if and only if $X$ is parallel.

Closed Riemannian manifolds do not admit, in general, globally defined parallel vector fields. Hence, one may expect that volume minimizing unit vector fields should be interesting for their symmetric properties. The following problem may be interesting.

Given a closed oriented Riemmannian manifold $M$ with vanishing Euler characteristic, what is the infimum (possibly a minimum) for the volume of all globally defined unit vector fields on $M$ ?

Perhaps, from a geometric viewpoint, the first closed Riemannian spaces to be studied are constant curvature spaces. In this class, the simplest ones are the flat tori and the unit round spheres of odd dimension. Closed spaces with constant negative curvature would come next.

Herman Gluck and Wolfgang Ziller proved the following result.

Theorem ([GZ]). The unit vector fields of minimum volume on $\boldsymbol{S}^{3}$ are precisely the Hopf vector fields and no others.

Here, Hopf vector fields on $\mathbf{S}^{3}$ are unit vector fields tangent to classical Hopf fibrations. Hopf vector fields $V_{H}$ can be defined on any $\mathbf{S}^{2 k+1}$ and it is easy to see, for example [GZ], that their volume is

$$
\operatorname{vol}\left(V_{H}\right)=2^{k} \operatorname{vol}\left(\mathbf{S}^{2 k+1}\right) \text {. }
$$

Later on, David Johnson showed

Theorem ([J]). Hopf fibrations on the round $\boldsymbol{S}^{5}$ are not local minima of the volume functional.

In fact, the method used in this proof may be extended to any $\mathbf{S}^{2 k+1}, k \geq 2$. See also [GmLf] for the instability of Hopf flows on spheres of radius $r$.

So, the problem of minimizing volume functional is open on spheres of odd dimension higher than 3 .

A very interesting example of unit vector field defined on $\mathbf{S}^{n}$ minus one point was given by Sharon Pedersen $[\mathrm{P}]$. We will denote this vector field by $V_{P}$. The volume of $V_{P}$ was calculated in $[\mathrm{P}]$ and, on the odd-spheres $\mathbf{S}^{2 k+1}$, is

$$
\operatorname{vol}\left(V_{P}\right)=\sqrt{2 \pi k} \operatorname{vol}\left(\mathbf{S}^{2 k+1}\right) .
$$


Note that this volume is much smaller than the volume of Hopf field.

Finally, we should mention that in the case of odd dimensional spheres $\mathbf{S}^{2 k+1}$, $k \geq 2$, H. Gluck and W. Ziller announced in [GZ] that a lower bound for the volume of unit vector fields should be

$$
\sum_{i=0}^{k} \frac{\left(\begin{array}{c}
k \\
i
\end{array}\right)^{2}}{\left(\begin{array}{c}
2 k \\
2 i
\end{array}\right)} \operatorname{vol}\left(\mathbf{S}^{2 k+1}\right) \text {. }
$$

In this paper we prove the following Theorem.

Main Theorem. Let $X$ be a unit vector field on a compact Riemannian and oriented manifold $M^{2 k+1}$. Then

$$
\operatorname{vol}(X) \geq \int_{M}\left(1+\sum_{i=1}^{k}\left(\begin{array}{c}
k \\
i
\end{array}\right)\left(\begin{array}{c}
2 k \\
2 i
\end{array}\right)^{-1}\left|\sigma_{2 i}\left(X^{\perp}\right)\right|\right) \nu,
$$

where $\sigma_{2 i}\left(X^{\perp}\right)$ is the $2 i$-th elementary symmetric function of the second fundamental form of the distribution orthogonal to $X$ (that is not necessarily integrable). When $k \geq 2$, equality holds if and only if $X$ is totally geodesic and $X^{\perp}$ is integrable and umbilic.

When $M$ has constant sectional curvature, we may apply an integral formula for the symmetric functions $\sigma_{2 i}$, see $[\mathrm{A}]$ or $[\mathrm{BLR}]$, to get the following

Corollary 1. Let $X$ be a unit vector field on $\boldsymbol{S}^{2 k+1}$ then

$$
\operatorname{vol}(X) \geq \sum_{i=0}^{k} \frac{\left(\begin{array}{c}
k \\
i
\end{array}\right)^{2}}{\left(\begin{array}{c}
2 k \\
2 i
\end{array}\right)} \operatorname{vol}\left(\boldsymbol{S}^{2 k+1}\right) \text {. }
$$

Note that there are no totally geodesic one dimensional foliations of $\mathbf{S}^{2 k+1}$ such that the normal bundle be integrable (see Section 5). So, the last point of Main Theorem implies

Corollary 2. There is no unit vector field on $\boldsymbol{S}^{2 k+1}, k \geq 2$, with volume equal to $\sum_{i=0}^{k} \frac{\left(\begin{array}{l}k \\ i\end{array}\right)^{2}}{\left(\begin{array}{c}2 k \\ 2 i\end{array}\right)} \operatorname{vol}\left(\boldsymbol{S}^{2 k+1}\right)$.

Let $V_{R}$ be a radial vector field on $\mathbf{S}^{2 k+1} \backslash\{ \pm p\}$, that is, a totally geodesic vector field obtained by means of the exponential map of $\mathbf{S}^{2 k+1}$ at $p$. The field $V_{R}$ is such that its normal bundle is integrable and its leaves are umbilical submanifolds of $\mathbf{S}^{2 k+1} \backslash\{ \pm p\}$.

Direct computations of the volume of a radial vector field $V_{R}$ on $\mathbf{S}^{2 k+1}$ with two antipodal singularities leads to 
Corollary 3. Let $X$ be any non-singular unit vector field on $\boldsymbol{S}^{2 k+1}$, then

$$
\operatorname{vol}\left(V_{R}\right) \leq \operatorname{vol}(X)
$$

The value of the bound in Corollary 1, which is the volume of the radial field, was estimated by Pedersen in $[\mathrm{P}]$ and she shown that

$$
\operatorname{vol}\left(V_{R}\right) \approx \sqrt{\pi k} \operatorname{vol}\left(\mathbf{S}^{2 k+1}\right)
$$

Then, the volumes of the mentioned vector fields on $\mathbf{S}^{2 k+1}$, when $k \geq 2$, hold the inequality

$$
\operatorname{vol}\left(\mathbf{S}^{2 k+1}\right)<\operatorname{vol}\left(V_{R}\right)<\operatorname{vol}\left(V_{P}\right) \ll \operatorname{vol}\left(V_{H}\right) .
$$

Finally, applying Main Theorem with [BLR] to spaces of negative constant curvature one gets

Corollary 4. Let $M^{2 k+1}$ be a compact, oriented, Riemannian manifold with constant sectional curvature $c<0$. Let $X$ be a unit vector field on $M$. Then,

$$
\operatorname{vol}(X) \geq \sum_{i=0}^{k} \frac{\left(\begin{array}{c}
k \\
i
\end{array}\right)^{2}|c|^{i}}{\left(\begin{array}{c}
2 k \\
2 i
\end{array}\right)} \operatorname{vol}(M) .
$$

We should remark that Corollary 1 confirms the lower bound announced for the volume on unit vector fields on odd dimensional spheres appearing in [GZ].

Also, Corollary 2 brings some light to a comment appearing in $[\mathrm{J}]$ (see page 927 , line -9) about whether certain calibration on $T^{1} \mathbf{S}^{2 k+1}$ is tangent to the graph of a flow on $\mathbf{S}^{2 k+1}$.

The paper is organized as follows. In Section 2 we expound some algebraic details that we will use afterwards. In Section 3, we present in the Fundamental Lemma a basic formula for the volume of a diagonal matrix (the Main Theorem when $X^{\perp}$ is integrable). In Section 4 we analyze the reduction of an arbitrary vector field to one with $X^{\perp}$ integrable. And finally, in Section 5, we prove the Main Theorem and its corollaries.

\section{Algebraic preliminaries}

The volume of a linear transformation $T: V^{n} \rightarrow V^{n}$ is the volume of the graph of the cube under $T$. That is, if $\varphi: V \rightarrow V \times V$ is given by $\varphi(v)=(v, T v)$, then

$$
\operatorname{vol}(T)=\operatorname{vol}(\varphi(V)) .
$$

In order to calculate the volume of $\varphi(V)$ we do the following. We define the application

$$
\begin{aligned}
\left.\varphi_{*}: V \times \stackrel{(n)}{ } \times\right) & \longrightarrow \Lambda^{n}(V \times V) \\
\left(v_{1}, \ldots, v_{n}\right) & \mapsto \varphi\left(v_{1}\right) \wedge \cdots \wedge \varphi\left(v_{n}\right)
\end{aligned}
$$


which is multilinear and anti-symmetric. By the universal property, $\varphi_{*}$ defines a unique application that we denote by the same letter, $\varphi_{*}: \Lambda^{n} V \rightarrow \Lambda^{n}(V \times V)$, such that

$$
\varphi_{*}\left(v_{1} \wedge \cdots \wedge v_{n}\right)=\varphi\left(v_{1}\right) \wedge \cdots \wedge \varphi\left(v_{n}\right) .
$$

By definition, for an orthonormal basis $\left\{e_{i}\right\}_{i=1}^{n}$ of $V$

$$
\operatorname{vol}(T)=\operatorname{vol}(\varphi(V))=\left\|\varphi_{*}\left(e_{1} \wedge \cdots \wedge e_{n}\right)\right\|=\left\|\varphi\left(e_{1}\right) \wedge \cdots \wedge \varphi\left(e_{n}\right)\right\| .
$$

This expression is evidently independent of the orthonormal basis chosen.

Proposition. Let $T$ be an endomorphism and $A=\left(a_{i j}\right)$ the matrix of $T$ associated to some orthonormal basis. Then,

$$
\begin{gathered}
\operatorname{vol}(T)=\left(1+\sum_{1 \leq i, j \leq n} a_{i j}^{2}+\sum_{\substack{i_{1}<i_{2} \\
j_{1}<j_{2}}}\left(\operatorname{det} A_{i_{1} i_{2}}^{j_{1} j_{2}}\right)^{2}+\ldots\right. \\
\left.\ldots+\sum_{\substack{i_{1}<\cdots<i_{n-1} \\
j_{1}<\cdots<j_{n-1}}}\left(\operatorname{det} A_{i_{1} \ldots i_{n-1}}^{j_{1} \ldots j_{n-1}}\right)^{2}+(\operatorname{det} A)^{2}\right)^{\frac{1}{2}},
\end{gathered}
$$

where $A_{i_{1} \ldots i_{k}}^{j_{1} \ldots j_{k}}$ is the submatrix of $A$ corresponding to the rows $\left(i_{1} \ldots i_{k}\right)$ and the columns $\left(j_{1} \ldots j_{k}\right)$.

Proof. For simplicity, let us denote the basis of $V \times V$ by $\left\{e_{i}=\left(e_{i}, 0\right)\right.$, $\left.e_{n+i}=\left(0, e_{i}\right)\right\}_{i=1}^{n}$. The $n$-vector $\varphi_{*}\left(e_{1} \wedge \cdots \wedge e_{n}\right)$ is:

$$
\varphi\left(e_{1}\right) \wedge \cdots \wedge \varphi\left(e_{n}\right)=\left(e_{1}+\sum_{i=1}^{n} a_{i 1} e_{n+i}\right) \wedge \cdots \wedge\left(e_{n}+\sum_{i=1}^{n} a_{i n} e_{n+i}\right) .
$$

Now we want to express the $n$-vector $\varphi\left(e_{1}\right) \wedge \cdots \wedge \varphi\left(e_{n}\right)$ in the natural basis of $\Lambda^{n}(V \times V),\left\{e_{i_{1}} \wedge \cdots \wedge e_{i_{n}} \mid 1 \leq i_{1}<\cdots<i_{n} \leq 2 n\right\}$. Performing the product in the last equation we get

$$
\begin{aligned}
& \varphi\left(e_{1}\right) \wedge \cdots \wedge \varphi\left(e_{n}\right)=e_{1} \wedge \cdots \wedge e_{n}+ \\
& \quad+\sum_{1 \leq i, j \leq n} a_{i j} e_{1} \wedge \cdots \wedge e_{j-1} \wedge e_{n+i} \wedge e_{j+1} \wedge \cdots \wedge e_{n}+ \\
& \quad+\sum_{\substack{i_{1}<i_{2} \\
j_{1}<j_{2}}}\left(a_{i_{1} j_{1}} a_{i_{2} j_{2}}-a_{i_{1} j_{2}} a_{i_{2} j_{1}}\right) e_{1} \wedge \cdots \wedge e_{j_{1}-1} \wedge e_{n+i_{1}} \wedge e_{j_{1}+1} \wedge \cdots \\
& \quad \cdots \wedge e_{j_{2}-1} \wedge e_{n+i_{2}} \wedge e_{j_{2}+1} \wedge \cdots \wedge e_{n}+\cdots \\
& \cdots+\sum_{\substack{i_{1}<\cdots<i_{n-1} \\
j_{1}<\cdots<j_{n-1}}}(-1)^{j_{n}-1} \operatorname{det}\left(A_{i_{1} \ldots i_{n-1}}^{j_{1} \ldots j_{n-1}}\right) e_{j_{n}} \wedge e_{n+i_{1}} \wedge \cdots \wedge e_{n+i_{n-1}}+ \\
& \quad+\operatorname{det}(A) e_{n+1} \wedge \cdots \wedge e_{2 n},
\end{aligned}
$$


where, in the last sum, $j_{n}$ is the only element of $\{1, \ldots, n\} \backslash\left\{j_{1}, \ldots\right.$, $\left.j_{n-1}\right\}$ and $A_{i_{1} \ldots i_{k}}^{j_{1} \ldots j_{k}}$ is the submatrix of $A$ corresponding to the rows $\left(i_{1} \ldots i_{k}\right)$ and the columns $\left(j_{1} \ldots j_{k}\right)$.

Then, the coefficients of $\varphi\left(e_{1}\right) \wedge \cdots \wedge \varphi\left(e_{n}\right)$ in the basis of $\Lambda^{n}(V \times V)$ are all the minor determinants of $A$, and also the 1 correspondent to $e_{1} \wedge \cdots \wedge e_{n}$. With this, we can calculate $\left\|\varphi\left(e_{1}\right) \wedge \cdots \wedge \varphi\left(e_{n}\right)\right\|^{2}$ adding the squares of each coefficient, as we have proclaimed in (3).

For simplicity, from now on we will call volume of the matrix $A$ to the right side of (3). If we change the orthonormal basis in $V$ obviously the volume of $A$ will remain the same but not each minor which appears in (3). For this reason, we will try to obtain another similar matrix $B=P A P^{-1}$ in such a way that we can calculate the volume of the endomorphism easily.

This change of basis in $V$ will represent an orthonormal change of frame in $M^{2 k+1}$ when we will identify $A$ with the second fundamental form of the distribution $X^{\perp}$.

\section{Volume of a diagonal matrix}

Fundamental Lemma. Let $D$ be a diagonal matrix of dimension $2 k$ with positive entries. We have

$$
\operatorname{vol}(D)^{2} \geq\left(1+\sum_{i=1}^{k} \frac{\left(\begin{array}{c}
k \\
i
\end{array}\right)}{\left(\begin{array}{c}
2 k \\
2 i
\end{array}\right)} \sigma_{2 i}(D)\right)^{2}
$$

where $\sigma_{2 i}(D)$ are the elementary symmetric functions of order $2 i$ of the elements of the diagonal. The equality holds if and only if $D=\lambda \operatorname{Id}_{2 k}$.

Proof. If the components of the matrix $D$ are $\left\{\lambda_{1}, \ldots, \lambda_{2 k}\right\}$, it is easy to see that the square of the volume of $D$ (the sum of the squares of all the minors) expressed in $(3)$ is

$$
\operatorname{vol}(D)^{2}=1+\sum_{i=1}^{2 k} \lambda_{i}^{2}+\ldots+\sum_{i_{1}<\cdots<i_{2 k-1}}\left(\lambda_{i_{1}}^{2} \cdots \lambda_{i_{2 k-1}}^{2}\right)+\lambda_{1}^{2} \cdots \lambda_{2 k}^{2} .
$$

We can span the square of the right side of (4) to obtain:

$$
\begin{aligned}
& \left(1+\sum_{i=1}^{k} \frac{\left(\begin{array}{l}
k \\
i
\end{array}\right)}{\left(\begin{array}{c}
2 k \\
2 k
\end{array}\right)} \sigma_{2 i}(D)\right)^{2}=1+\sum_{i=1}^{k} \frac{\left(\begin{array}{l}
k \\
i
\end{array}\right)^{2}}{\left(\begin{array}{c}
2 k \\
2 i
\end{array}\right)^{2}} \sigma_{2 i}^{2}+\sum_{i=1}^{k} 2 \frac{\left(\begin{array}{c}
k \\
i
\end{array}\right)}{\left(\begin{array}{c}
2 k \\
2 i
\end{array}\right)} \sigma_{2 i}+ \\
& +\sum_{1 \leq i<j \leq k} 2 \frac{\left(\begin{array}{l}
k \\
i
\end{array}\right)\left(\begin{array}{c}
k \\
j
\end{array}\right)}{\left(\begin{array}{c}
2 k \\
2 i
\end{array}\right)\left(\begin{array}{c}
2 k \\
2 j
\end{array}\right)} \sigma_{2 i} \sigma_{2 j}=\sum_{j=0}^{2 k} \sum_{i=0}^{j} \frac{\left(\begin{array}{c}
k \\
i
\end{array}\right)\left(\begin{array}{c}
k \\
j-i
\end{array}\right)}{\left(\begin{array}{c}
2 k \\
2 i
\end{array}\right)\left(\begin{array}{c}
2 k \\
2 j-2 i
\end{array}\right)} \sigma_{2 i} \sigma_{2 j-2 i} .
\end{aligned}
$$

Recall that 


$$
\sigma_{j}(D)=\sigma_{j}=\sum_{i_{1}<\cdots<i_{j}} \lambda_{i_{1}} \cdots \lambda_{i_{j}}
$$

that $\sigma_{0}=1$ and, if $j>2 k$, then $\sigma_{j}=0$.

Inequality (4) is basically a relation between polynomials in the variables $\left\{\lambda_{i}\right\}$ and then we must compare the terms of the same degree on each side of the inequality.

The last equality of (6) groups the different terms of degree $2 j$. That is, in

$$
\left(1+\sum_{i=1}^{k} \frac{\left(\begin{array}{c}
k \\
i
\end{array}\right)}{\left(\begin{array}{c}
2 k \\
2 i
\end{array}\right)} \sigma_{2 i}(D)\right)^{2}
$$

the terms of degree $2 j$ in the $\lambda_{i}$ are exactly

$$
\sum_{i=0}^{j} \frac{\left(\begin{array}{c}
k \\
i
\end{array}\right)\left(\begin{array}{c}
k \\
j-i
\end{array}\right)}{\left(\begin{array}{c}
2 k \\
2 i
\end{array}\right)\left(\begin{array}{c}
2 k \\
2 j-2 i
\end{array}\right)} \sigma_{2 i} \sigma_{2 j-2 i} .
$$

From (5) and (6), in order to prove the Lemma, we have to show that:

$$
\sum_{i_{1}<\cdots<i_{j}} \lambda_{i_{1}}^{2} \cdots \lambda_{i_{j}}^{2} \geq \sum_{i=0}^{j} \frac{\left(\begin{array}{c}
k \\
i
\end{array}\right)\left(\begin{array}{c}
k \\
j-i
\end{array}\right)}{\left(\begin{array}{c}
2 k \\
2 i
\end{array}\right)\left(\begin{array}{c}
2 k \\
2 j-2 i
\end{array}\right)} \sigma_{2 i} \sigma_{2 j-2 i}
$$

for $j=1, \ldots, 2 k$.

Note that in the sum of the right hand several terms appear twice (in fact, all the terms or all except one depending on the parity of $j$ ).

From the well-known inequality $\left(a_{1}^{2}+a_{2}^{2}+\ldots+a_{n}^{2}\right) \geq \frac{1}{n}\left(a_{1}+a_{2}+\ldots+a_{n}\right)^{2}$ we get

$$
\sum_{i_{1}<\cdots<i_{j}} \lambda_{i_{1}}^{2} \cdots \lambda_{i_{j}}^{2} \geq \frac{1}{\left(\begin{array}{c}
2 k \\
j
\end{array}\right)}\left(\sum_{i_{1}<\cdots<i_{j}} \lambda_{i_{1}} \cdots \lambda_{i_{j}}\right)^{2}=\frac{1}{\left(\begin{array}{c}
2 k \\
j
\end{array}\right)} \sigma_{j}^{2} .
$$

In order to compare $\sigma_{j}^{2}$ with the products $\sigma_{2 i} \sigma_{2 j-2 i}$ we use the formula in [HLP, pg. 52]. This formula is valid when the $\lambda_{i}$ 's are positive. If we normalize the symmetric functions we have

$$
p_{i}=\frac{1}{\left(\begin{array}{c}
2 k \\
i
\end{array}\right)} \sigma_{i}
$$

and then, see [HLP]

$$
p_{i}^{2} \geq p_{i-1} p_{i+1} \quad i=1, \ldots, 2 k-1 .
$$

In (9), equality holds if and only if all $\lambda_{i}$ 's have the same value. From this equation we can obtain easily

$$
p_{j}^{2} \geq p_{j-2} p_{j+2},
$$

and consecutively we get 


$$
\sigma_{j}^{2} \geq \frac{\left(\begin{array}{c}
2 k \\
j
\end{array}\right)^{2}}{\left(\begin{array}{c}
2 k \\
j-2 s
\end{array}\right)\left(\begin{array}{c}
2 k \\
j+2 s
\end{array}\right)} \sigma_{j-2 s} \sigma_{j+2 s} \quad \text { for all possible } j \text { and } s .
$$

We show here the proof of the case $j$ even. The other case is completely similar. Assume that $j$ is even. From (8) and (10) we have, for all $s=0, \ldots, j / 2$,

$$
\sum_{i_{1}<\cdots<i_{j}} \lambda_{i_{1}}^{2} \cdots \lambda_{i_{j}}^{2} \geq \frac{\left(\begin{array}{c}
2 k \\
j
\end{array}\right)}{\left(\begin{array}{c}
2 k \\
j-2 s
\end{array}\right)\left(\begin{array}{c}
2 k \\
j+2 s
\end{array}\right)} \sigma_{j-2 s} \sigma_{j+2 s} .
$$

In order to obtain the coefficient of $\sigma_{j-2 s} \sigma_{j+2 s}$ in the general expression (7), we use the previous inequality to obtain

$$
2 \frac{\left(\begin{array}{c}
k \\
\frac{j}{2}-s
\end{array}\right)\left(\begin{array}{c}
k \\
\frac{j}{2}+s
\end{array}\right)}{\left(\begin{array}{c}
2 k \\
j
\end{array}\right)} \sum_{i_{1}<\cdots<i_{j}} \lambda_{i_{1}}^{2} \cdots \lambda_{i_{j}}^{2} \geq 2 \frac{\left(\begin{array}{c}
k \\
\frac{j}{2}-s
\end{array}\right)\left(\begin{array}{c}
k \\
\frac{j}{2}+s
\end{array}\right)}{\left(\begin{array}{c}
2 k \\
j-2 s
\end{array}\right)\left(\begin{array}{c}
2 k \\
j+2 s
\end{array}\right)} \sigma_{j-2 s} \sigma_{j+2 s}
$$

for $s=1, \ldots, j / 2$ and when $s=0$,

$$
\frac{\left(\begin{array}{c}
k \\
j / 2
\end{array}\right)^{2}}{\left(\begin{array}{c}
2 k \\
j
\end{array}\right)} \sum_{i_{1}<\cdots<i_{j}} \lambda_{i_{1}}^{2} \cdots \lambda_{i_{j}}^{2} \geq \frac{\left(\begin{array}{c}
k \\
j / 2
\end{array}\right)^{2}}{\left(\begin{array}{c}
2 k \\
j
\end{array}\right)^{2}} \sigma_{j}^{2} .
$$

The case $s=0$ is not different from the others $(s>0)$ when $j$ is odd. Therefore, to show (7) we must see that the sum of the coefficients of (11) for all $s=1, \ldots, j / 2$ with the coefficient of (12) is exactly 1 . That is, we have to prove that

$$
2 \sum_{s=1}^{j / 2} \frac{\left(\begin{array}{c}
k \\
\frac{j}{2}-s
\end{array}\right)\left(\begin{array}{c}
k \\
\frac{j}{2}+s
\end{array}\right)}{\left(\begin{array}{c}
2 k \\
j
\end{array}\right)}+\frac{\left(\begin{array}{c}
k \\
j / 2
\end{array}\right)^{2}}{\left(\begin{array}{c}
2 k \\
j
\end{array}\right)}=1,
$$

or, equivalently,

$$
\left(\begin{array}{c}
2 k \\
j
\end{array}\right)=2 \sum_{l=0}^{j / 2-1}\left(\begin{array}{c}
k \\
l
\end{array}\right)\left(\begin{array}{c}
k \\
j-l
\end{array}\right)+\left(\begin{array}{c}
k \\
j / 2
\end{array}\right)^{2}=\sum_{l=0}^{j}\left(\begin{array}{c}
k \\
l
\end{array}\right)\left(\begin{array}{c}
k \\
j-l
\end{array}\right) .
$$

But this equality holds because both combinatorial expressions can be calculated from the expansion $(1+x)^{2 k}$ directly as well as from $\left((1+x)^{k}\right)^{2}$ in this way:

$$
\begin{aligned}
& \sum_{j=0}^{2 k}\left(\begin{array}{c}
2 k \\
j
\end{array}\right) x^{j}=(1+x)^{2 k}=(1+x)^{k}(1+x)^{k}= \\
& =\left(\sum_{q_{1}=0}^{k}\left(\begin{array}{c}
k \\
q_{1}
\end{array}\right) x^{q_{1}}\right)\left(\sum_{q_{2}=0}^{k}\left(\begin{array}{c}
k \\
q_{2}
\end{array}\right) x^{q_{2}}\right)=\sum_{j=0}^{2 k} \sum_{q_{1}+q_{2}=j}\left(\begin{array}{c}
k \\
q_{1}
\end{array}\right)\left(\begin{array}{c}
k \\
q_{2}
\end{array}\right) x^{q_{1}+q_{2}}= \\
& =\sum_{j=0}^{2 k} \sum_{l=0}^{j}\left(\begin{array}{l}
k \\
l
\end{array}\right)\left(\begin{array}{c}
k \\
j-l
\end{array}\right) x^{j} .
\end{aligned}
$$


The coefficients of $x^{j}$ in both sides of the this equality give (13).

Finally, we remark that in this the proof, as well the proof of the odd case, the inequalities become equalities if and only if all $\lambda_{i}$ 's are equal, that is, when $\lambda=\lambda_{1}=\lambda_{2}=\ldots=\lambda_{2 k}$.

The Fundamental Lemma provides an inequality when $\lambda_{i} \geq 0$ where $\left\{\lambda_{1}, \ldots \lambda_{2 k}\right\}$ are the components of the diagonal matrix $D$. If we follow carefully the proof we can observe that the expression can be a little more general. If $D$ is an arbitrary diagonal matrix with elements $\lambda_{i} \in \mathbb{R}$ we get

$$
\operatorname{vol}(D) \geq \sum_{i=0}^{k} \frac{\left(\begin{array}{c}
k \\
i
\end{array}\right)}{\left(\begin{array}{c}
2 k \\
2 i
\end{array}\right)} \sigma_{2 i}\left(\left\{\left|\lambda_{j}\right|\right\}_{j=1}^{2 k}\right) \geq \sum_{i=0}^{k} \frac{\left(\begin{array}{c}
k \\
i
\end{array}\right)}{\left(\begin{array}{c}
2 k \\
2 i
\end{array}\right)}\left|\sigma_{2 i}\left(\left\{\lambda_{j}\right\}_{j=1}^{2 k}\right)\right| .
$$

\section{Reduction to the diagonal case}

A unit vector field $X$ on $M^{2 k+1}$ defines a, possibly non-integrable, distribution of dimension $2 k$, say $X^{\perp}$. At each point, the (non-symmetric) second fundamental form of this distribution has an associated endomorphism. Let $A$ be its matrix with respect to some basis. The eigenvalues of A can be real or complex numbers.

When all the eigenvalues are real, we have in fact that $X^{\perp}$ is integrable. In this case the endomorphism is symmetric and hence we can choose an orthonormal local basis who diagonalizes A. When $X^{\perp}$ is not integrable, we can assure at least, through an orthonormal change of frame, that there exists a local basis in which the matrix associated is of type

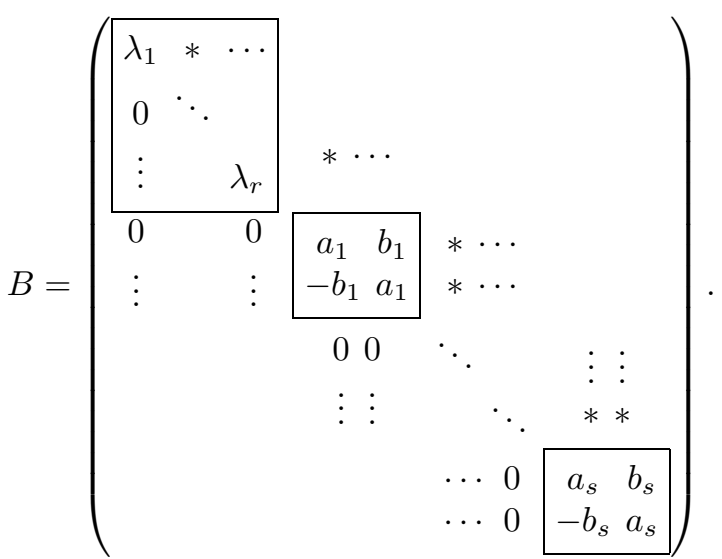

That is, $B$ is a matrix with an upper triangular part (the $\lambda_{i} \mathrm{~s}$ ) and another part composed with $2 \times 2$ submatrices along the diagonal. The components under the diagonal are all zero and the components above the diagonal are not important. The $\lambda_{i}$ 's represent the real eigenvalues and the $\left(\begin{array}{cc}a_{i} & b_{i} \\ -b_{i} & a_{i}\end{array}\right)$ the complex ones. 
With the aim to prove the Main Theorem for the general case, we will need several steps in order to reach the diagonal case (when $X^{\perp}$ is integrable). Along these consecutive transformations we must control the volume of the matrices and also the values of the $\sigma_{2 i}$ that appear in the lower bound of (2).

Lemma 1. From a generic matrix $A$ and the matrix $B$ described above, we have,

$$
\operatorname{vol}(A)=\operatorname{vol}(B) \quad \text { and } \quad \sigma_{i}(A)=\sigma_{i}(B) \quad \text { for all } i .
$$

Proof. The matrices $A$ and $B$ represent the same endomorphism but for different orthonormal bases. Hence $B=P A P^{-1}$ for some orthogonal matrix $P$ and we have directly

$$
\operatorname{vol}(A)=\operatorname{vol}(B)
$$

By definition, the elementary symmetric functions $\sigma_{i}$ satisfy the equation

$$
\operatorname{det}(A-t \mathrm{Id})=\sum_{i=0}^{n}(-1)^{i} \sigma_{n-i}(A) t^{i} .
$$

But,

$$
\begin{gathered}
\operatorname{det}(A-t \mathrm{Id})=\operatorname{det}\left(P(A-t \mathrm{Id}) P^{-1}\right)=\operatorname{det}\left(P A P^{-1}-t \mathrm{Id}\right)= \\
=\operatorname{det}(B-t \mathrm{Id})=\sum_{i=0}^{n}(-1)^{i} \sigma_{n-i}(B) t^{i} .
\end{gathered}
$$

Then, $\sigma_{i}(A)=\sigma_{i}(B)$ for all $i=1, \ldots, n$.

From $B$, we make another matrix $C$ taking just the real eigenvalues as well as the $2 \times 2$ boxes corresponding to the complex eigenvalues. We fill in with zeros the rest of the matrix. That is, we define

$$
C=\left(\begin{array}{cccccccccc}
\lambda_{1} & 0 & \ldots & & & & & & \\
0 & \ddots & & & & & & & \\
\vdots & & \lambda_{r} & 0 & 0 & \ldots & & & \\
& \ldots & 0 & a_{1} & b_{1} & 0 & \ldots & & \\
& & \vdots & -b_{1} & a_{1} & 0 & \ldots & & \\
& & & & & \ddots & & \vdots & \vdots \\
& & & & & & \ddots & 0 & 0 \\
& & & & & & \ddots & 0 & \\
& & & & & \cdots & 0 & a_{s} & b_{s} \\
& & & & & \cdots & 0 & -b_{s} & a_{s}
\end{array}\right) .
$$

The endomorphism associated to $C$ is possibly quite different from that of $B$. This construction is completely artificial but we obtain the following. 
Lemma 2. If $B$ and $C$ are the matrices described above, then

$$
\operatorname{vol}(B) \geq \operatorname{vol}(C) \quad \text { and } \quad \sigma_{i}(B)=\sigma_{i}(C) \quad \text { for all } i .
$$

The volumes will be equal if and only if $B=C$.

Proof. In order to calculate the volume of $B$ we need the squares of all the minor determinants. The value of some of these determinants form the volume of $C$ while the rest provides the inequality. These remaining determinants are formed with the entries above the diagonal. For this, in $\operatorname{vol}(B) \geq \operatorname{vol}(C)$ we will get the equality when all the components above the diagonal be zero, that is, when $B=C$.

It is also trivial that $\sigma_{i}(B)=\sigma_{i}(C)$ for all $i=1, \ldots n$. The $\sigma_{i}$ is just the sum of all the diagonal determinants of order $i$. The matrices $B$ and $C$ have the same components in the diagonal and under the diagonal all the elements are zero. Thus the diagonal determinants calculated with entries in $B$ or in $C$ will have the same value.

From $C$, we take the absolute values of the elements of the diagonal and we define $\widetilde{C}$.

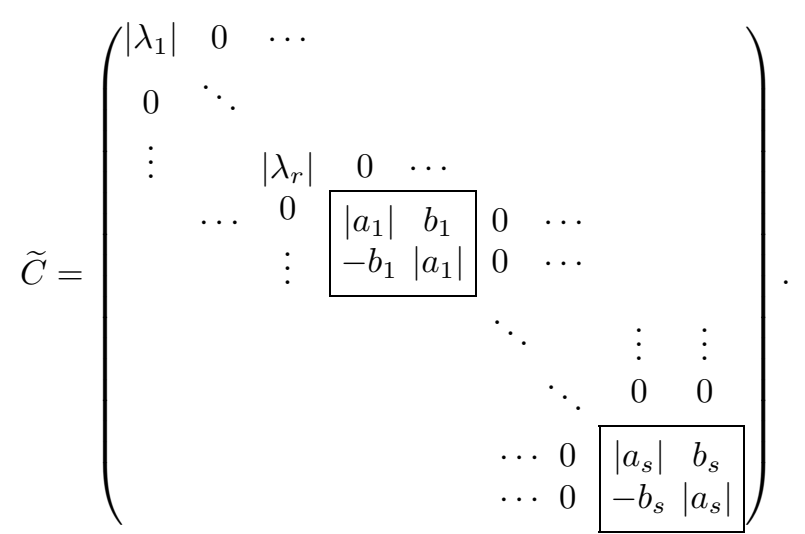

It is straightforward from the definition that

$$
\operatorname{vol}(C)=\operatorname{vol}(\widetilde{C}) \text { and }\left|\sigma_{2 i}(C)\right| \leq \sigma_{2 i}(\widetilde{C}) \text { for all } i .
$$


Finally, from $\widetilde{C}$ we construct the diagonal matrix $D$,

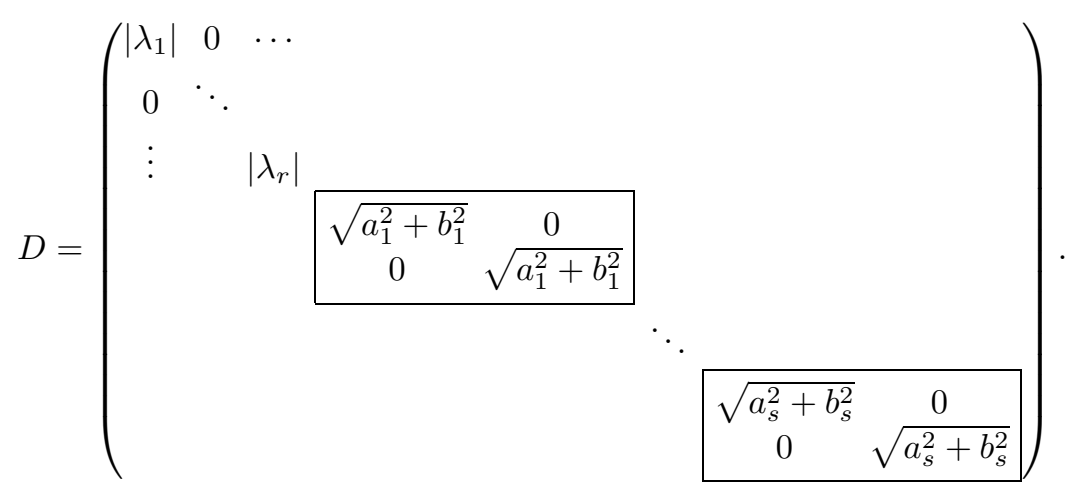

Lemma 3. Let $\widetilde{C}$ and $D$ be as described above. Then we have

$$
\operatorname{vol}(\widetilde{C})=\operatorname{vol}(D) \quad \text { and } \quad \sigma_{i}(\widetilde{C}) \leq \sigma_{i}(D) \quad \text { for all } i .
$$

When the dimension is greater or equal than 4 , the $\sigma_{i}$ 's inequality will be an equality if and only if $\widetilde{C}=D$, (that is, $b_{j}=0$ for all $j$ ).

Proof. In order to improve the notation, let us assume that the $\lambda_{i}$ and the $a_{j}$ are all positive avoiding the need to write $\left|\lambda_{i}\right|$ or $\left|a_{j}\right|$.

In order to obtain $\operatorname{vol}(\widetilde{C})$, if we calculate the determinants of order 1 we note that the possible differences with $\operatorname{vol}(D)$ appear in the components $\left(\begin{array}{cc}a_{i} & b_{i} \\ -b_{i} & a_{i}\end{array}\right)$. In this case, the squares of the determinants of order 1 will add up

$$
2 a_{i}^{2}+2 b_{i}^{2} .
$$

For the corresponding elements in $D,\left(\begin{array}{cc}\sqrt{a_{i}^{2}+b_{i}^{2}} & 0 \\ 0 & \sqrt{a_{i}^{2}+b_{i}^{2}}\end{array}\right)$, we get the same value

$$
\left(\sqrt{a_{i}^{2}+b_{i}^{2}}\right)^{2}+\left(\sqrt{a_{i}^{2}+b_{i}^{2}}\right)^{2}=2 a_{i}^{2}+2 b_{i}^{2}
$$

For the determinants of order 2 , in $\widetilde{C}$ we have

$$
\operatorname{det}^{2}\left(\begin{array}{cc}
a_{i} & b_{i} \\
-b_{i} & a_{i}
\end{array}\right)=\left(a_{i}^{2}+b_{i}^{2}\right)^{2}
$$

but in $D$ we have also

$$
\operatorname{det}^{2}\left(\begin{array}{cc}
\sqrt{a_{i}^{2}+b_{i}^{2}} & 0 \\
0 & \sqrt{a_{i}^{2}+b_{i}^{2}}
\end{array}\right)=\left(a_{i}^{2}+b_{i}^{2}\right)^{2} .
$$

When we look at the determinants of order 2 that separate the entries of the $2 \times 2$ boxes in $\widetilde{C}$, we found the following results: 


$$
\begin{array}{rlrl}
2 \operatorname{det}^{2}\left(\begin{array}{cc}
\lambda_{i} & 0 \\
0 & a_{j}
\end{array}\right) & =2 \lambda_{i}^{2} a_{j}^{2}, & 2 \operatorname{det}^{2}\left(\begin{array}{cc}
\lambda_{i} & 0 \\
0 & b_{j}
\end{array}\right) & =2 \lambda_{i}^{2} b_{j}^{2}, \\
4 \operatorname{det}^{2}\left(\begin{array}{cc}
a_{i} & 0 \\
0 & a_{j}
\end{array}\right) & =4 a_{i}^{2} a_{j}^{2}, & 4 \operatorname{det}^{2}\left(\begin{array}{cc}
a_{i} & 0 \\
0 & b_{j}
\end{array}\right) & =4 a_{i}^{2} b_{j}^{2}, \\
4 \operatorname{det}^{2}\left(\begin{array}{cc}
b_{i} & 0 \\
0 & a_{j}
\end{array}\right) & =4 a_{j}^{2} b_{i}^{2}, & 4 \operatorname{det}^{2}\left(\begin{array}{cc}
b_{i} & 0 \\
0 & b_{j}
\end{array}\right)=4 b_{i}^{2} b_{j}^{2} .
\end{array}
$$

But in $D$ we get the same values:

$$
\begin{aligned}
2 \operatorname{det}^{2}\left(\begin{array}{cc}
\lambda_{i} & 0 \\
0 & \sqrt{a_{j}^{2}+b_{j}^{2}}
\end{array}\right) & =2 \lambda_{i}^{2}\left(a_{j}^{2}+b_{j}^{2}\right), \\
\operatorname{ddet}^{2}\left(\begin{array}{cc}
\sqrt{a_{i}^{2}+b_{i}^{2}} & 0 \\
0 & \sqrt{a_{j}^{2}+b_{j}^{2}}
\end{array}\right) & =4\left(a_{i}^{2}+b_{i}^{2}\right)\left(a_{j}^{2}+b_{j}^{2}\right) .
\end{aligned}
$$

We can observe that this argument is valid for the determinants of any order. A full proof can be given for the general case. We have presented here the kernel of the proof and we prefer to save the reader the boring details. Thus we can assert

$$
\operatorname{vol}(\widetilde{C})=\operatorname{vol}(D)
$$

In order to check the inequality of the $\sigma_{i}$ 's, we remark that the diagonal determinants that do not separate the $2 \times 2$ boxes corresponding to the complex eigenvalues are exactly the same in $\widetilde{C}$ that in $D$. Just the diagonal determinants in $\widetilde{C}$ that take only one $a_{i}$ and the equivalent in $D$ that take just one root $\sqrt{a_{i}^{2}+b_{i}^{2}}$ are equal if and only if $b_{i}=0$ (if $a_{i}>0$ ). Then,

$$
\sigma_{i}(\widetilde{C}) \leq \sigma_{i}(D) \text { for all } i=1, \ldots, n \text {. }
$$

We can observe that in dimension 2 or 3 it can happen $\sigma_{2}(D)=\sigma_{2}(\widetilde{C})$ but with $b_{1} \neq 0$. When $n \geq 4$, we will have that $\sigma_{i}(\widetilde{C}) \leq \sigma_{i}(D)$ is an equality just when $b_{i}=0$ for all $i \quad(\widetilde{C}=D)$.

Redefining the elements of $D$, after several steps, we have reduced a general matrix $A$ to a diagonal one,

$$
D=\left(\begin{array}{cccc}
\lambda_{1} & 0 & \cdots & 0 \\
0 & \ddots & & \\
\vdots & & & \\
0 & & & \lambda_{2 k}
\end{array}\right)
$$

where all $\lambda_{i}$ are positive. 


\section{Proof of the Main Theorem and consequences}

Proof of Main Theorem. Let $X$ be a unit vector field and $\left\{e_{1}, e_{2}, \ldots e_{2 k}\right.$, $\left.e_{2 k+1}=X\right\}$ a local frame on $M^{2 k+1}$. Let A be the matrix of the second fundamental form of the complementary distribution to $X$ (possibly non-integrable). The matrix A defines an endomorphism of the subspace $X_{x}^{\perp} \subset T_{x} M$ for all $x \in M$. From an algebraic viewpoint, $X_{x}^{\perp}$ is a vector space of dimension $2 k$ endowed with a scalar product.

From the expression of the volume of a unit field (1), we omit the terms where the acceleration $\nabla_{X} X$ is involved. Thus,

$$
\operatorname{vol}(X) \geq \int_{M} \operatorname{vol}(\mathrm{A}) \nu
$$

where equality holds if and only if $X$ is totally geodesic $\left(\nabla_{X} X=0\right)$.

Now we recall the modifications described in Section 4 . These transformations represent, at each point of $M$, a change of the second fundamental form endomorphism of $X^{\perp}$.

Through an orthonormal change of basis in $X^{\perp}$ we can modify the matrix $A$ as we have described in Lemma 1 obtaining in this way an upper triangular matrix $B$ with maybe $2 \times 2$ boxes in the diagonal. The matrix $C$ introduced before Lemma 2 is nearly diagonal, with the exception of the $2 \times 2$ diagonal blocks. Taking the absolute values of the entries of the diagonal we define $\widetilde{C}$. Finally, let $D$ be the diagonal matrix like in Lemma 3 (with positive components). Thus,

$$
\int_{M} \operatorname{vol}(\mathrm{A}) \nu=\int_{M} \operatorname{vol}(B) \nu \geq \int_{M} \operatorname{vol}(C) \nu=\int_{M} \operatorname{vol}(\widetilde{C}) \nu=\int_{M} \operatorname{vol}(D) \nu .
$$

Also by Lemmas 1, 2 and 3 and from (14) we have the inequalities

$$
\sigma_{2 i}(D) \geq \sigma_{2 i}(\widetilde{C}) \geq\left|\sigma_{2 i}(C)\right|=\left|\sigma_{2 i}(B)\right|=\left|\sigma_{2 i}(\mathrm{~A})\right| .
$$

Thus, using (15), (16), Fundamental Lemma and (17) consecutively,

$$
\begin{aligned}
\operatorname{vol}(X) & \geq \int_{M} \operatorname{vol}(D) \nu \geq \int_{M}\left(1+\sum_{i=1}^{k} \frac{\left(\begin{array}{c}
k \\
i
\end{array}\right)}{\left(\begin{array}{c}
2 k \\
2 i
\end{array}\right)} \sigma_{2 i}(D)\right) \nu \geq \\
& \geq \int_{M}\left(1+\sum_{i=1}^{k} \frac{\left(\begin{array}{c}
k \\
i
\end{array}\right)}{\left(\begin{array}{c}
2 k \\
2 i
\end{array}\right)}\left|\sigma_{2 i}(\mathrm{~A})\right|\right) \nu,
\end{aligned}
$$

as we have stated in (2).

When $k \geq 2$, the inequalities of Lemmas 2 and 3 will be equalities if and only if the endomorphism $A$ is diagonalizable (that is, all the eigenvalues are real). The Fundamental Lemma says that in order to have an equality all the eigenvalues must be equal. Thus, equality in (2) will be satisfied if and only if $X$ is totally geodesic and $X^{\perp}$ is integrable and umbilic, $\mathrm{A}=\lambda \operatorname{Id}_{2 k}$.

As we mentioned before, the integrals of the $\sigma_{2 i}\left(X^{\perp}\right)$ in manifolds of constant sectional curvature have been calculated in [BLR]. These integrals turn out to be 
independent on the field $X$. In this way, in $\mathbf{S}^{2 k+1}$ and also in compact hyperbolic spaces the lower bound of Main Theorem is actually a lower bound of the volume functional.

Theorem ([BLR]). Let $M^{n+1}$ be a closed manifold with constant sectional curvature $c$ and $X$ a unit vector field. Then,

$$
\int_{M} \sigma_{i}\left(X^{\perp}\right) \nu= \begin{cases}\left(\begin{array}{c}
n / 2 \\
i / 2
\end{array}\right) c^{i / 2} \operatorname{vol}(M) & \text { if } n \text { and } i \text { even } \\
0 & \text { if } n \text { or } i \text { odd. }\end{cases}
$$

In this way, the proof of Corollary 1 is straightforward. In $[\mathrm{P}]$ we find developed the value of this sum and it can be checked that

$$
\sum_{i=0}^{k} \frac{\left(\begin{array}{c}
k \\
i
\end{array}\right)^{2}}{\left(\begin{array}{c}
2 k \\
2 i
\end{array}\right)}=\frac{4^{k}}{\left(\begin{array}{c}
2 k \\
k
\end{array}\right)}
$$

Then, we can rewrite the lower bound of Corollary 1 in this way

$$
\operatorname{vol}(X) \geq \frac{4^{k}}{\left(\begin{array}{c}
2 k \\
k
\end{array}\right)} \operatorname{vol}\left(\mathbf{S}^{2 k+1}\right) .
$$

When we try to look for globally defined unit vector fields on $\mathbf{S}^{2 k+1}$ satisfying minimization conditions of the Main Theorem we find in the mathematical literature the following result.

Theorem ([BW]). Let $M$ be a complete Riemannian manifold with non-negative Ricci curvature. If $\mathcal{F}$ is a foliation of codimension 1 over $M$ and $\mathcal{F}^{\perp}$, the normal flow to $\mathcal{F}$, is geodesic, then $\mathcal{F}$ is totally geodesic and $\mathcal{F}^{\perp}$ is parallel. Therefore, $M$ is locally a Riemannian product.

Proof of Corollary 2. If there were on $\mathbf{S}^{2 k+1}$ a vector field $X$ who reached the lower bound of Corollary $1, X$ would be totally geodesic with orthogonal distribution, $X^{\perp}$, also integrable. With this and the previous Theorem showed in [BW], the sphere would be a Riemannian product. Contradiction.

Proof of Corollary 3. The distribution orthogonal to $V_{R}$ in $M=\mathbf{S}^{2 k+1} \backslash\{ \pm p\}$ is in fact a foliation and the integral leaves are the parallels of $\mathbf{S}^{2 k+1}$. From (1) we have

$$
\operatorname{vol}\left(V_{R}\right)=\int_{M}\left(\sum_{i=0}^{2 k}\left(\begin{array}{c}
2 k \\
i
\end{array}\right) \lambda^{2 i}\right)^{\frac{1}{2}} \nu=\int_{M}\left(\left(1+\lambda^{2}\right)^{2 k}\right)^{\frac{1}{2}} \nu=\int_{M}\left(1+\lambda^{2}\right)^{k} \nu,
$$

where $\lambda$ is the principal curvature of the leaf. It is well-known that the radius $r$ of a parallel of the sphere is given by

$$
\left(\frac{1}{r}\right)^{2}=1+\lambda^{2}
$$


If we parametrize the sphere $\mathbf{S}^{2 k+1}$ by the latitude $\theta$, then $r=\cos (\theta)$ and

$$
\begin{aligned}
\operatorname{vol}\left(V_{R}\right) & =\int_{M}\left(1+\lambda^{2}\right)^{k} \nu=\int_{-\frac{\pi}{2}}^{\frac{\pi}{2}} \int_{\mathbf{S}^{2 k}(\cos (\theta))}\left(\frac{1}{\cos ^{2}(\theta)}\right)^{k} \nu^{\prime} d \theta= \\
& =\int_{-\frac{\pi}{2}}^{\frac{\pi}{2}} \frac{1}{\cos ^{2 k}(\theta)} \operatorname{vol}\left(\mathbf{S}^{2 k}(\cos (\theta))\right) d \theta=\int_{-\frac{\pi}{2}}^{\frac{\pi}{2}} \frac{2 \pi^{\frac{2 k+1}{2}}}{\Gamma\left(\frac{2 k+1}{2}\right)} d \theta= \\
& =\frac{2^{2 k} \pi^{\frac{2 k+3}{2}}(k-1) !}{(2 k-1) ! \sqrt{\pi}}=\frac{2^{2 k+1} \pi^{k+1} k !}{(2 k) !}=\frac{4^{k}}{\left(\begin{array}{c}
2 k \\
k
\end{array}\right)} \frac{2 \pi^{k+1}}{k !}= \\
& =\frac{4^{k}}{\left(\begin{array}{c}
2 k \\
k
\end{array}\right)} \operatorname{vol}\left(\mathbf{S}^{2 k+1}\right) .
\end{aligned}
$$

We have remarked before that $4^{k}\left(\begin{array}{c}2 k \\ k\end{array}\right)^{-1}$ is equal to the sum expressed in the Corollary 1 . This complete the proof.

The proof of Corollary 4 is also direct from Main Theorem.

\section{References}

[A] D. Asimov, Average Gaussian curvatures of leaves of foliations, Bull. Amer. Math. Soc. 84 (1978), 131-134.

[BLR] F. G. B. Brito, R. Langevin and H. Rosenberg, Intégrales de courbure sur des variétés feuilletées, J. Differential Geom. 16 (1981), 19-50.

[BW] F. G. B. Brito and P. G. Walczak, Totally geodesic foliation with integrable normal bundles, Bol. Soc. Brasil. Mat. 17, (1986), 41-46.

[GmLf] O. Gil-Medrano and E. Llinares-Fuster, Second variation of volume and energy of vector fields. Stability of Hopf vector fields, Math. Ann. 320 (2001), 531-545.

[GZ] H. Gluck and W. Ziller, On the volume of the unit vector fields on the three sphere, Comment Math. Helv. 61 (1986), 177-192.

[HLP] G. H. Hardy, J. E. Littlewood and G. Pólya, Inequalities, Cambridge University Press, 1988.

[J] D. L. Johnson, Volume of flows, Proc. Amer. Math. Soc. 104 (1988), 923-932.

[P] S. L. Pedersen, Volumes of vector fields on spheres, Trans. Amer. Math. Soc. 336 (1993), 69-78. 
F. B. Brito

Dpto. de Matemática

Instituto de Matemática e Estatística

Universidade de São Paulo

R. do Matão 1010

São Paulo-SP

05508-090

Brazil

e-mail: fabiano@ime.usp.br

A. M. Naveira

Dpto. de Geometría y Topología

Facultad de Matemáticas

Universidad de Valencia

Avda. Vicente Andrés Estellés no 1

46100 Burjassot (Valencia)

Spain

e-mail: naveira@uv.es

(Received: August 12, 2002)
P. M. Chacón

Dpto. Matemática Aplicada

Facultad de Informática

Universidad de Murcia

Campus Universitario de Espinardo

30100 Murcia

Spain

e-mail: pmchacon@um.es

(1) To access this journal online:

(ID) http://www.birkhauser.ch 